\title{
Phenomics for photosynthesis, growth and reflectance in Arabidopsis thaliana reveals circadian and long-term fluctuations in heritability
}

Pádraic J. Flood ${ }^{1,2,5}$, Willem Kruijer ${ }^{3}$, Sabine K. Schnabel ${ }^{3}$, Rob van der Schoor ${ }^{4,6}$, Henk Jalink ${ }^{4,6}$, Jan F. H. Snel ${ }^{4,7}$, Jeremy Harbinson ${ }^{2}$ and Mark G. M. Aarts ${ }^{1 *}$ (D)

\begin{abstract}
Background: Recent advances in genome sequencing technologies have shifted the research bottleneck in plant sciences from genotyping to phenotyping. This shift has driven the development of phenomics, high-throughput non-invasive phenotyping technologies.

Results: We describe an automated high-throughput phenotyping platform, the Phenovator, capable of screening 1440 Arabidopsis plants multiple times per day for photosynthesis, growth and spectral reflectance at eight wavelengths. Using this unprecedented phenotyping capacity, we have been able to detect significant genetic differences between Arabidopsis accessions for all traits measured, across both temporal and environmental scales. The high frequency of measurement allowed us to observe that heritability was not only trait specific, but for some traits was also time specific.

Conclusions: Such continuous real-time non-destructive phenotyping will allow detailed genetic and physiological investigations of the kinetics of plant homeostasis and development. The success and ultimate outcome of a breeding program will depend greatly on the genetic variance which is sampled. Our observation of temporal fluctuations in trait heritability shows that the moment of measurement can have lasting consequences. Ultimately such phenomic level technologies will provide more dynamic insights into plant physiology, and the necessary data for the omics revolution to reach its full potential.
\end{abstract}

Keywords: Photosynthesis, Phenomics, Chlorophyll fluorescence, Heritability, High throughput screening, Natural variation, Arabidopsis thaliana

\section{Background}

Photosynthesis is the primary entry point of energy into the biosphere and as such provides the foundation for life on earth. One prominent class of photosynthetic organisms are plants, which are responsible for the vast majority of the energy and biomass influx in the terrestrial biosphere. They are also the basis of our economy,

\footnotetext{
*Correspondence: mark.aarts@wur.nl

1 Laboratory of Genetics, Wageningen University, Wageningen,

The Netherlands

Full list of author information is available at the end of the article
}

providing the majority of calories necessary to sustain humanity. It is clear that plant photosynthesis is the keystone for our existence, but we know surprisingly little about the extent and basis of variation in this most fundamental of traits [14]. The overarching reason for our lack of knowledge about intraspecific variation in photosynthesis is our inability to efficiently screen large numbers of plants. This epistemic Rubicon must be overcome for our survival, as photosynthesis is the only major productivity-related trait which has yet to be improved [30]. To facilitate this, high-throughput phenotyping of photosynthesis must be developed. 
Obtaining phenotypic data is the most time consuming and labour intensive step of many biological experiments [21]. Despite this, the detail and extent of phenotypic data compares poorly with the increasingly complete genotype data now available [13, 22, 37]. This is not only due to the recent advances in genomics but also due to the complex multidimensional nature of phenotypes [21]. The vast number of phenotypic states that a genotype can occupy can be visualised as its phenotypic space, which is often referred to as its phenome. In practice the phenome is a theoretical entity which can never be fully characterised. This was recognised by Houle et al. [22] leading them to propose that phenomics may be understood as the "acquisition of high dimensional phenotypic data on an organism wide scale".

The phenotype is the result of the interplay between genetics and developmental, environmental and stochastic influences, where the intensity, frequency, order and interaction of these influences affect the outcome. Traditionally, due to its labour intensive nature, phenotyping was only feasible for a single time point on a subset of the traits which comprise the phenome. To reveal, however, the dynamic and variable nature of the phenome, requires numerous measurements across developmental and environmental gradients [21]. Some phenomic (in the sense of Houle et al.) quality datasets for endophenotypes, i.e. transcriptomics, metabolomics, proteomics, ionomics, lipidomics, and even RNA directly undergoing translation (translatomics) have been produced [24, 25]. But as they rely on destructive measurements they only provide a snapshot of the endophenome at the time of measurement. These omics datasets not only lack dynamic insight but they also fail in another import aspect: they give no information about fluxes or growth. Yet, kinetic phenotypes or functional states, such as growth or photosynthesis, provide the most direct and integrative quantification of plant performance [24]. They represent the combined effect of all other phenotypic levels, so the relevance of, for example, variation in gene expression can be assessed at higher organizational levels.

Both photosynthesis and plant growth are ideal traits to assess the functional relevance of endophenotypic omics datasets. Plant size reflects the integration of metabolic and developmental processes and is a good indicator of long term performance whilst photosynthesis and growth rate reflect more immediate physiological responses $[9,12]$. Growth responses are most dynamic at the meristematic level [26] which is not amenable to rapid, frequent measurement, in contrast to plant size and photosynthesis, which together provide an ideal phenotypic window into genotype performance.

To this end we set about developing a high-throughput phenotyping platform which would allow us to continuously phenotype a large number of plants for photosynthesis and growth. This will result in phenomic data, though we recognise that the full characterisation of the phenome, namely all possible phenotypic outcomes, across all levels of organisational, developmental and environmental space, is beyond our current capabilities. Nevertheless this is a valuable step forward and will give high-dimensional phenotypic data which, in accordance with Houle et al. [22] can be considered phenomics.

High intensity screening of a particular trait will allow for temporally detailed estimation of heritability. Broad sense heritability is a measure of how much of the phenotypic variance in a population can be attributed to genetic variation rather than other factors, such as a nonuniform environment [42]. It is often used to assess the potential responsiveness of traits to selection, whether natural or artificial $[28,31]$. Whilst it is well recognised that heritability is trait, population and environment specific, its variation with time is less well studied. If heritability shows significant time dependence then this will be of interest to breeders and evolutionary biologists, as the time point at which selection occurs will be crucial in determining the selection response. The ability to measure traits multiple times per day for prolonged periods in order to better understand the time-dependency of heritability was an important factor in the design of the phenotyping system we describe here.

\section{System development \\ Design considerations}

While the rationale on designing the phenotyping system is described here, the actual experimental conditions and mathematical approaches used to analyse the phenotype data we collected are described in the "Methods" section.

The overarching goal of the phenotyper system, which we named Phenovator, was accurate quantification of the phenotypic variation, so as to estimate the genetic variation, in natural populations of Arabidopsis thaliana, using photosynthesis and growth as phenotypic indicators of plant performance. This required that any noise, whether technical, environmental or otherwise, be minimized so that the genetic signal could be accurately assessed. This is particularly important when dealing with traits like photosynthesis, which are environmentally responsive and exhibit limited phenotypic diversity within a species [14]. Thus the plant growth environment should be well controlled to minimize heterogeneity of the environment and allow high reproducibility. Key environmental variables which have a large effect on plant performance and often elicit a phenotypic response, are light, water, temperature and nutrient availability. To control these inputs the Phenovator was located in a climate-controlled 
growth chamber and equipped with an automated watering system.

To allow repeated measurements of the same plants, and minimize any measurement effects, the measurements must be non-invasive. We therefore developed an image-based phenotyping platform. A balance also had to be struck between the extensive (range) and intensive (detail) capabilities of the Phenovator. We chose to measure a restricted set of phenotypes that are important indicators for plant performance (photosynthetic activity, size, and colour) and to measure these with a high frequency, opting for intensity of measurement. Our optical measurement system was based on a camera. Other camera-based phenotyping systems have been developed and in many the plants are moved to the phenotyping equipment $[2,23,39,40]$. This has the advantage that the number of plants that can be screened is only limited by the growing area, but the disadvantage is that the plants are not assayed under growth conditions and that the rate of throughput is decreased. In our system we opted to move the camera to the plants and as the camera can be moved at $6 \mathrm{~m} \mathrm{~s}^{-1}$ (much faster than a plant can be safely moved) we can image plants with a high-frequency, but the total growing area that can be imaged is limited by the camera movement system.

Since many phenotypes show spatial heterogeneity (see Fig. 1 for an example), it was essential to image the entire above-ground part of the plant (roots are outside the scope of this phenotyper). Since our target species, A. thaliana (Arabidopsis), forms a rosette, which until flowering is relatively flat, this could be achieved using a single camera. To be able to identify and characterise genetic variation we needed sufficient throughput to screen populations suited for genetic mapping, such as recombinant inbred line (RIL) populations or genome wide association (GWA) panels. The latter populations usually consist of 300 or more genotypes [27] which with four replicates per genotype yields a minimum screening capacity of 1200 plants. To capture short-term changes in the phenotype it was decided that it should be possible to measure all plants within $60 \mathrm{~min}$. Finally it was essential that the entire system was automated, with control and data storage outside the growth room to minimize environmental fluctuations (particularly carbon dioxide) due to people entering the room.

\section{System design}

The Phenovator we designed consists of five main parts: a supporting frame, an ebb and flood hydroponic system, an XY camera movement system, a camera and a computer to control camera movement, imaging and data storage (Fig. 2). The supporting frame was constructed from $100 \times 100 \mathrm{~mm}^{2}$ box-section aluminium beams (www.maytec.org) to support $\mathrm{X}-\mathrm{Y}$ rails and the basins in which plants were grown. It also provides the rigid, stable camera platform necessary for imaging. To be able to image quickly requires a platform that is sufficiently stiff to eliminate vibration after the camera movement is complete. The camera movement system (www.elmekanic.nl) is capable of speeds of $6 \mathrm{~m} \mathrm{~s}^{-1}$ (though for safety reasons this is currently limited to only $1 \mathrm{~m} \mathrm{~s}^{-1}$ ) and allows high reproducibility of camera positioning. We use a so-called a

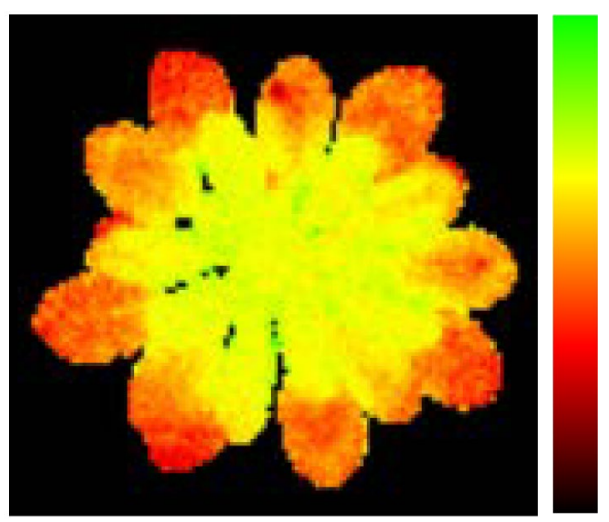

b

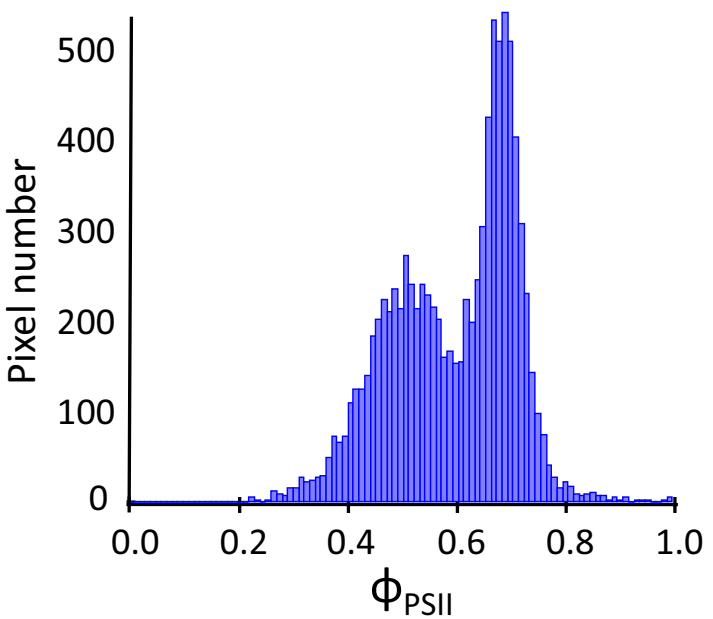

Fig. 1 Distribution of photosystem II efficiency (DPSII) in a phosphate deficient Arabidopsis. a False colour (DPSII image of a phosphate deficient plant, the scale bar on the right shows DPSII values from 0 (black) to 1 (green). b Image a plotted as a histogram of pixels at specific ФPSII values. The distribution is bimodal hence the mean value fails to aptly represent the plant's phenotype 

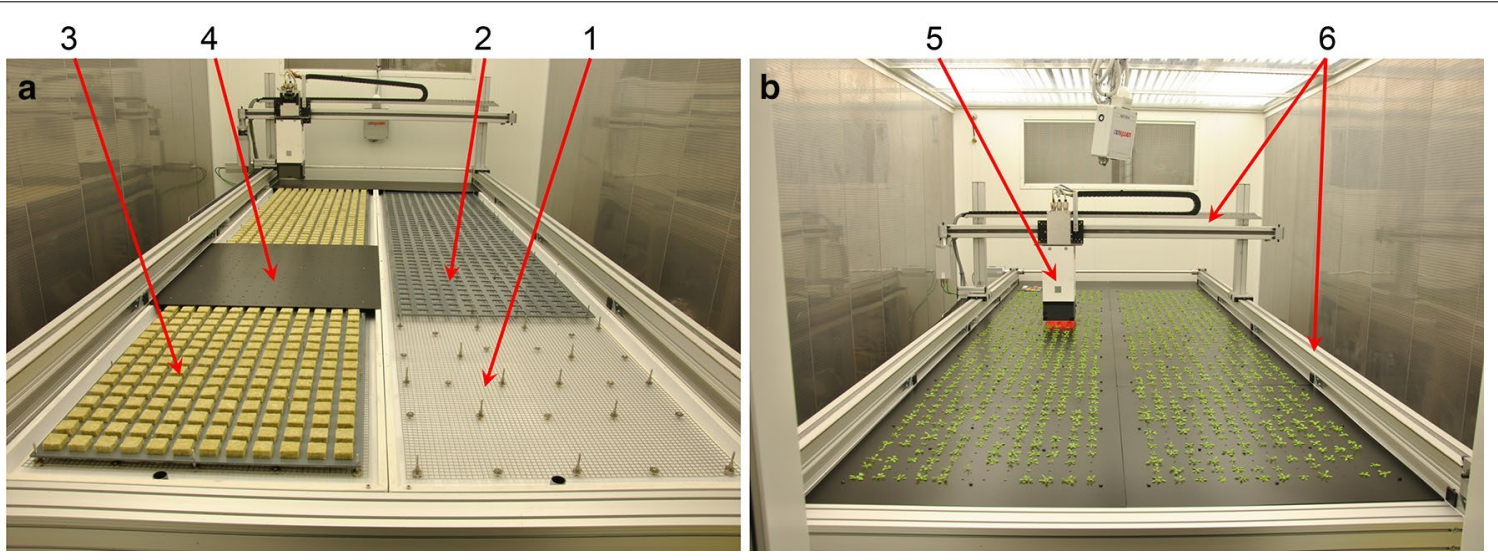

Fig. 2 The Phenovator. a The set-up of the growth system. 1 Support grid for the rockwool blocks with support pins for the rockwool block spacing plate and the top plate, 2 the rockwool block spacing plate to position and hold the blocks, 3 this grid with rockwool blocks in place, and 4 the black PVC top plate. $\mathbf{b}$ The Phenovator system in action: 5 the imaging head carrying the camera (the red light is the saturating pulse for DPSII measurement), 6 the $X-Y$ rails used to position the imaging head

"ebb and flood" hydroponic irrigation system to water and feed the plants growing in a rockwool (www.grodan. com) substrate (Fig. 2a). Rockwool is a synthetic, relatively inert, fibrous substrate which allows manipulation of plant nutrition regimes [18].

The growth system (Fig. 2) is comprised of two irrigation basins, each with their own irrigation tank and pump, which allows for two different irrigation and nutrient regimes to be applied in the same experiment. Each basin has space for 720 rockwool blocks $40 \times 40 \times 40 \mathrm{~mm}$ in size giving a total capacity of 1440 rockwool blocks. The rockwool blocks are held $20 \mathrm{~mm}$ apart by a PVC grid that is attached to a rigid stainless steel grid upon which the blocks rest. The PVC grid prevents any sideways movement of the rockwool blocks which could cause the plants to shear, and is held $15 \mathrm{~mm}$ above the stainless steel base grid by spacers. The stainless steel grid provides structural integrity to the rockwool support system and is supported $5 \mathrm{~mm}$ above the bottom of the irrigation basin. The perforations in the grid allow for free circulation of nutrient solution, ensuring that all blocks receive irrigation for approximately the same amount of time. A spacing of $<5 \mathrm{~mm}$ between the stainless steel base and the irrigation basin was found to sometimes cause problems of root death, possibly due to trapping of nutrient solution and anaerobiosis. On top of the rockwool blocks there is a black plastic nonreflective sheet of foamed PVC, $3 \mathrm{~mm}$ thick (Figs. 2a, 4). In this sheet, 3-mm countersunk holes were drilled at distances of $60 \mathrm{~mm}$ and positioned above the centre of each rockwool block. All three layers are held in place using threaded stainless steel pins which were welded to the stainless steel grid. Four support studs fit into sockets drilled into the irrigation basin to hold this grid in a fixed position. All materials were tested for phytotoxicity and corrosion resistance, and were washed thoroughly before use. The black plastic cover ensures that there is no algal growth, restricts soil dwelling organisms such as the larvae of fungus gnats (Bradysia spp.) and minimises background noise in the images, making automated image processing much easier.

Images are recorded using a monochrome camera (Pike; www.alliedvisiontec.com) mounted on the $\mathrm{X}-\mathrm{Y}$ movement system. An eight-position filter wheel is mounted between the lens and the ccd chip of the camera to capture images in different wavelength bands. We measure reflectance at 480, 532, 550, 570, 660, 700, 750 and $790 \mathrm{~nm}$ with each filter having a full width at half maximum (FWHM) of $10 \mathrm{~nm}$; these narrow spectral wavelength measurements allow for estimation of a range of plant pigments. The reflection bands at 480, 570 and $660 \mathrm{~nm}$ are used to construct red, green and blue (RGB) colour images. Chlorophyll content (Chl) is estimated from reflectance $(\mathrm{R})$ at 700 and $790 \mathrm{~nm}$ after AA Gitelson et al. [20] $\mathrm{Chl}=\left(\mathrm{R}_{700}{ }^{-1}-\mathrm{R} 790^{-1}\right) \times \mathrm{R} 790$.

Projected leaf area (PLA) provides a good estimate of above ground biomass [29] and is estimated from near infrared (NIR) reflection at $790 \mathrm{~nm}$; this wavelength was chosen so the plants could be measured both day and night without disturbing the day-night cycle. Four NIR light emitting diodes (LED) with a FWHM of $40 \mathrm{~nm}$ and a maximum radiant power of $1 \mathrm{~W}$ per LED provide the $790 \mathrm{~nm}$ radiation. NIR measurements are taken every $3 \mathrm{~h}$ resulting in eight images per day.

We use chlorophyll fluorescence imaging to measure $\Phi_{\text {PSII }}$ (the light-use efficiency of PSII electron transport, also known as $\mathrm{Fq}^{\prime} / \mathrm{Fm}^{\prime}$, or $\left.\Delta \mathrm{F} / \mathrm{Fm}\right)[3,16,41]$ using a variation of the method of Genty and Meyer 
[17]. This method has the advantage of a good signal to noise ratio and has proved very suitable for our imaging conditions in which the unfiltered background irradiance is low owing to the shadowing effect of the imaging system. Measurements are made by illuminating the plants at the growth chamber actinic light level (200 or $550 \mu \mathrm{mol} \mathrm{m} \mathrm{m}^{-2} \mathrm{~s}^{-1}$ ) with a centre wavelength of $630 \mathrm{~nm}$ and a FWHM of $20 \mathrm{~nm}$ for $10 \mathrm{~s}$ followed by a $2 \mathrm{~s}$ saturating pulse of $5000 \mu \mathrm{mol} \mathrm{m}{ }^{-2} \mathrm{~s}^{-1}$ using LEDs attached to the Phenovator camera head. At the end of the $10 \mathrm{~s}$ of actinic light and prior to the saturating light, 24 images are taken and averaged to generate the Fs image. During the saturating light pulse six images are taken of which that with the highest signal is used for the $\mathrm{Fm}^{\prime}$ image. The LEDs are turned off after the saturating pulse and an additional 24 images are taken and averaged in order to generate a dark image to account for any background light from the fluorescent lamps in the growth chamber. A fluorescent target, applied as a rubber compound [purchased from Thorlabs (www.thorlabs.de), but since withdrawn from the market] that shows fluorescence over a wide range of wave lengths, is imaged at the beginning of each measurement sequence in order to provide a factor to correct the Fs and $\mathrm{Fm}^{\prime}$ measurements for the difference in light intensity used to produce the images [17]. The camera measurement scheme was programmed so that immediately neighbouring positions were skipped and returned to later, thus allowing time for any disturbance of adjacent plants by either an increase or decrease of their irradiance to dissipate. Thus the Phenovator comprises only four moving parts, the $\mathrm{X}$ movement system, the Y movement system (these both comprise of motors, drive belts and bearings), the filter wheel and the camera focus. This simplicity is a strong advantage when long term experiments are undertaken.

\section{Data processing}

The growth platform containing the 1440 plants is divided up into 120 imaging positions (Additional file 1: Figure S1) each of which contains 12 plants $(3 \times 4)$ thus each measurement cycle results in 120 images each containing 12 plants. Different measurement tasks (imaging $\Phi_{\text {PSII }}$, NIR reflectance or spectral imaging) can be programmed in a daily schedule, which is used over the entire experiment. Analysis software has been developed to convert raw images from the imaging system to images of physiological parameters (e.g. $\Phi_{\text {PSII }}$ ) or biochemical composition (e.g. chlorophyll content). Each image is matched to a table position, and the genotype planted at each position is provided via a comma separated (csv) file, thus enabling the image processing software to group images by genotype. Based on images containing 12 plants the analysis software (available upon request) calculates per replicate the parameters for each genotype. Each measurement protocol (e.g. measurement of $\Phi_{\text {PSII }}$ ) produces its own parameters, which are calculated from a selected area within the image using a mask derived from the desired plant. A grid of vertical and horizontal reference lines (shown in Additional file 1: Figure S1) is set by the user and provide the coordinates around which a box is drawn to select individual plants. A greyscale threshold (or mask), set by the user, is used to distinguish the plant from the background within this box. Twelve areas are defined and used to obtain a specific plant from the image. Only the pixels within the mask are used to estimate the phenotypic parameters.

All images (raw data and derived data) are stored, and the values of each phenotype are calculated per pixel. Both the pixel values and the averages over images are available to output in csv format. The spatial distribution of pixel data within any stored image can be shown (Fig. 1; Additional file 1: Figure S1). Since our plants were grown for only 4 weeks under non-stressful conditions there was no spatial variation in any parameter so we will not discuss this further.

\section{Results}

\section{System uniformity}

The (spatial) uniformity and (temporal) reproducibility of the system were assessed by estimating the magnitude of several design factors using a mixed model (see "Methods" section; Additional file 2: Appendix S1 for an overview of the experiments), which included random effects for genotype, experiment, basin, and table position (Additional file 3: Appendix S2; Additional file 4: Data S1, Additional file 5: Data S2). Using this model, genotypic means were calculated as the best linear unbiased estimators (BLUEs) for genotype. Spatial variability was modelled by row $(x)$ and column $(y)$ effects, as well as within image rows $x_{\text {within }}$ and columns $y_{\text {within }}$. While $x$ and $y$ modelled the coordinates across the whole platform, $x_{\text {within }}$ and $y_{\text {within }}$ modelled the spatial effects within images of 12 plants $(3 \times 4)$. In addition to the main effects, second and third order interactions between design factors were included. A more detailed description of all design factors is given in Additional file 3: Appendix S2.

For all traits and time points there is considerable genetic variation: the variance component for genotype is of a similar order of magnitude as the residual error variance, which is consistent with the heritability estimates found below. Although the main effect of experiment was substantial, the genotype by experiment interaction was negligible for almost all traits. Only for the spectral measurements at 700 and $750 \mathrm{~nm}$ the genotype by experiment interaction was larger, but still small 
compared to the main genotypic variance (Additional file 4: Data S1, Additional file 5: Data S2). The phenotypic ranking of the genotypes can therefore be expected to be consistent across experiments. For $\Phi_{\text {PSII }}$ and spectral measurements, the position within the image showed a considerable main effect which is likely due to light gradients in the camera head. This effect of position within the image showed no interaction with genotype (Additional file 4: Data S1, Additional file 5: Data S2) and thus could be corrected for. In a few cases, there was some interaction between experiment and within image position, but never with genotype. Table position and the $\mathrm{x}$ and $\mathrm{y}$ coordinate across the whole platform showed a small main effect for some of the spectral measurements. Nevertheless, the very low variances of the interactions between genotype, experiment and the design-effects indicate that we can combine data from different experiments, allowing phenotyping of potentially thousands of genotypes.

\section{Phenotypic variation}

The Phenovator has three main imaging protocols in routine use (Fig. 3). The first is used to measure photosynthetic efficiency via chlorophyll fluorescence $\left(\Phi_{\mathrm{PSII}}\right)$, the second is used to measure pigment content via spectral imaging and the third measures PLA via NIR imaging.

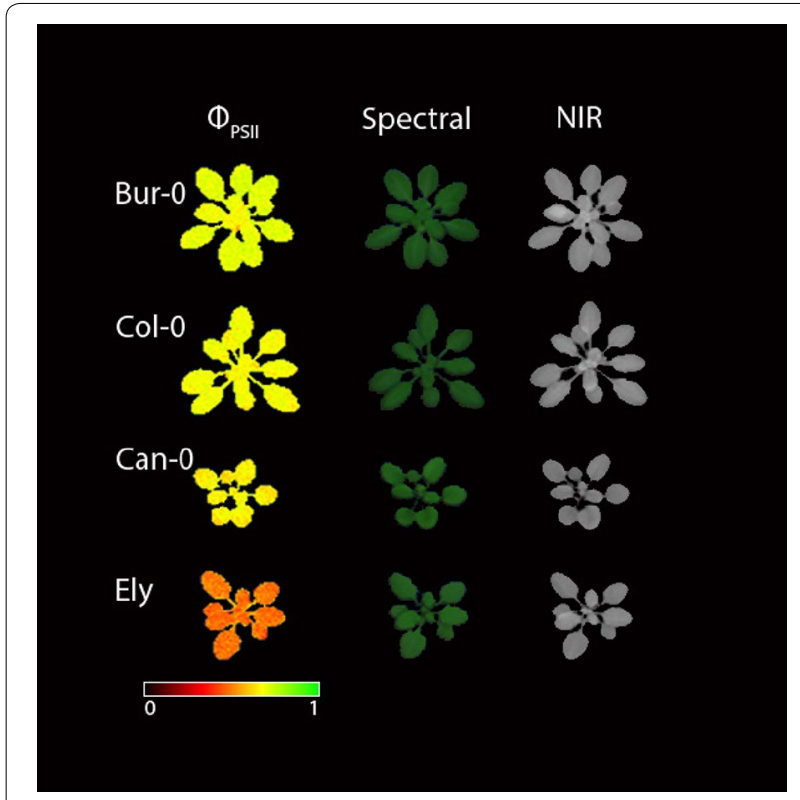

Fig. 3 Examples of images generated by the Phenovator. The first column shows false colour images of photosystem II efficiency (DPSII) running from 0 (black) to 1 (green). The second column shows the red-green-blue (RGB) output of the spectral measurements. The third column shows the images generated by near infrared imaging (NIR) at $790 \mathrm{~nm}$. The rows correspond to four different genotypes, accessions Bur-0, Col-0, Can-0 and Ely. Ely is atrazine resistant, hence the much lower ФPSII
Figure $4 \mathrm{a}, \mathrm{b}$ shows the total variation for $\Phi_{\mathrm{PSII}}$ for 20 genotypes grown at 200 and $550 \mu \mathrm{mol} \mathrm{m}{ }^{-2} \mathrm{~s}^{-1}$ light intensity. The two different light intensities were chosen both to test the flexibility of the system and to assess the response of the genotypes to these different conditions. $\Phi_{\text {PSII }}$ is influenced by both the light intensity and the genetic background of the plant measured. In addition to these differences, the high resolution measurements allow the observation of both a daily fluctuation in $\Phi_{\text {PSII }}$ as well as a gradual upward trend through time at the higher light intensity.

The two genotypes with the lowest $\Phi_{\mathrm{PSII}}$ are Ely, an atrazine resistant accession known to have a low light-use efficiency for PSII electron transport [11], and RIL BC354 from the Bur- $0 \times$ Col-0 population [38], which is known to carry a mutant version of the PDE237 gene affecting photosynthesis [43]. However, even without these unusual genotypes there is substantial variation for $\Phi_{\text {PSII }}$. The variation from approx. 0.62 to 0.72 at $200 \mu \mathrm{mol} \mathrm{m}^{-2} \mathrm{~s}^{-1}$ and 0.54 to 0.63 at $550 \mu \mathrm{mol} \mathrm{m} \mathrm{m}^{-2} \mathrm{~s}^{-1}$ is about $12 \%$ (assuming an upper limit of 0.8 for $\Phi_{\text {PSII }}$ ) for normal natural accessions, extending to almost $40 \%$ when the lines with unusually low light-use efficiency are included.

The chlorophyll reflectance index (Fig. 4c, d) is a linear measure of chlorophyll content and, as expected [1], decreases as the irradiance is increased from 200 to $550 \mu \mathrm{mol} \mathrm{m}{ }^{-2} \mathrm{~s}^{-1}$. The phenotypic variation in spectral reflectance at each wavelength can be observed, with an increase in light intensity having opposite effects on different wavelengths (Additional file 6: Figure S2). PLA can be measured at short intervals, allowing the construction of growth curves (Fig. 4e, f). Both genetic background and light intensity have a large effect on growth rates. Another interesting phenomenon is the undulating nature of the curves due to leaf movement. The percentage difference between images at neighbouring time points shows the movement more clearly (Fig. 4g, h). The plant growth and leaf movement phenotypes are easily revealed and analysed because of the high imaging frequency. The fluctuation in PLA due to leaf movement can result in negative apparent growth rates, so we smoothed the curves before estimating growth rates (Fig. 5).

\section{Genetic variation}

The heritability of a trait is a measure of the proportion of phenotypic variance explained by genetic effects [42]. Figure 6a shows the heritability through time for $\Phi_{\mathrm{PSII}}$. Addition of the photosynthetic extremes greatly inflates the genotypic variance which results in very high estimates of heritability. The heritability of $\Phi_{\mathrm{PSII}}$ also shows a slight but recurrent daily rise, but is not affected by the difference in light intensity. The heritability of chlorophyll reflectance index and PLA show more gradual 

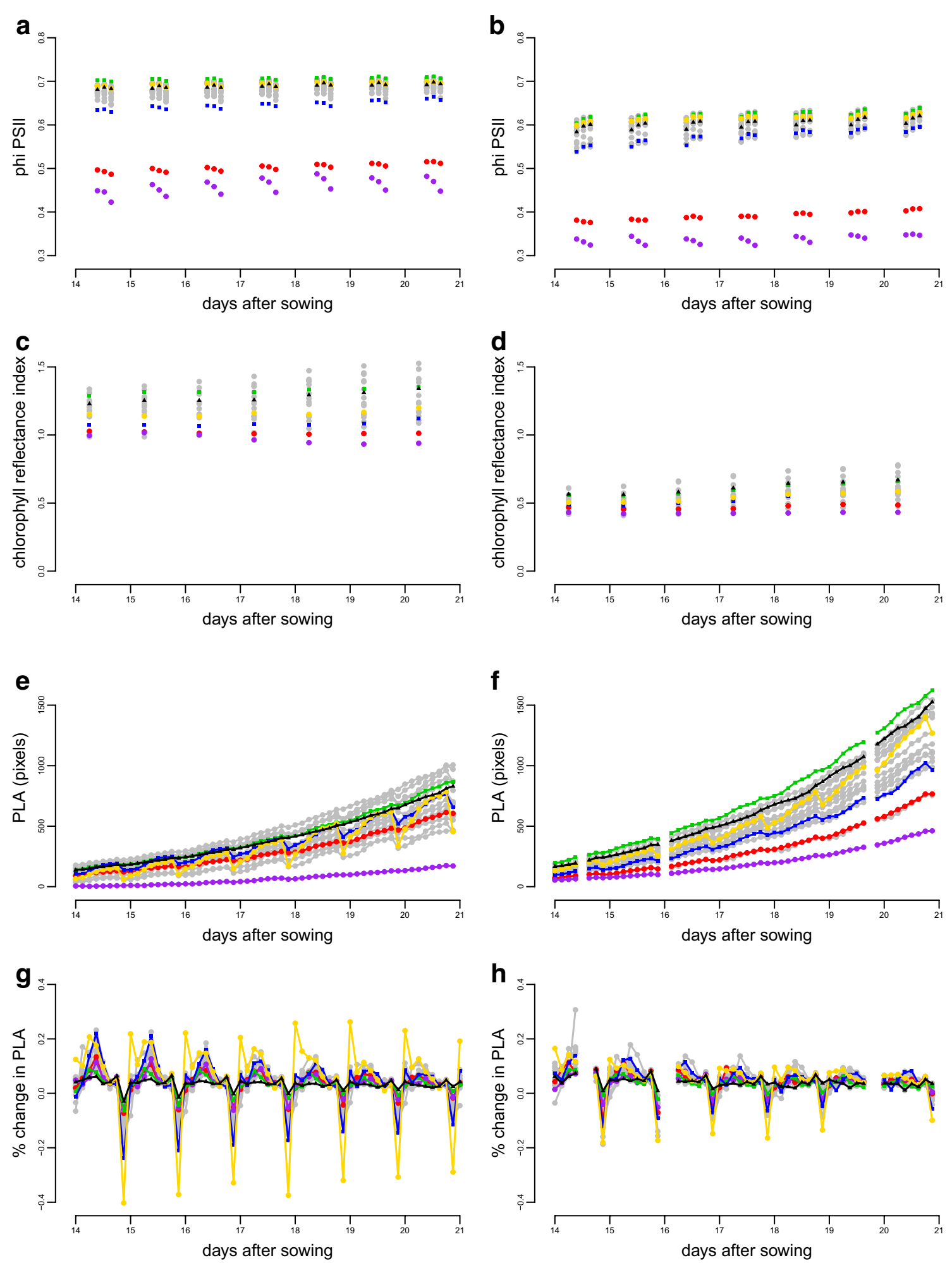

Fig. 4 Phenotypic diversity in twenty Arabidopsis genotypes grown at $200 \mu \mathrm{mol} \mathrm{m}{ }^{-2} \mathrm{~s}^{-1}$ light intensity (a, c, e, g), and $550 \mu \mathrm{mol} \mathrm{m}{ }^{-2} \mathrm{~s}^{-1}$ light intensity $(\mathbf{b}, \mathbf{d}, \mathbf{f}, \mathbf{h})$. Graphs $(\mathbf{a}, \mathbf{b})$ shows $\Phi_{\text {PSIII }}$ through time; $\mathbf{c}, \mathbf{d}$ shows chlorophyll reflectance index; $\mathbf{e}, \mathbf{f}$ shows projected leaf area (PLA). Finally, $\mathbf{g}$ $\mathbf{h}$ shows percentage change in PLA every $3 \mathrm{~h}$. All data points are genotypic means (BLUEs), combining observations on replicates from different experiments into one representative value for each genotype at each time point. Six genotypes, An-2 (yellow circles), BC354 (purple circles), Bur-0 (green squares), Col-0 (black triangles), Ely (red circles) and Ts-1 (blue squares) are indicated in colour. Error bars have been excluded for clarity, the significance of between genotype differences is apparent from the heritability estimates in Fig. 6 


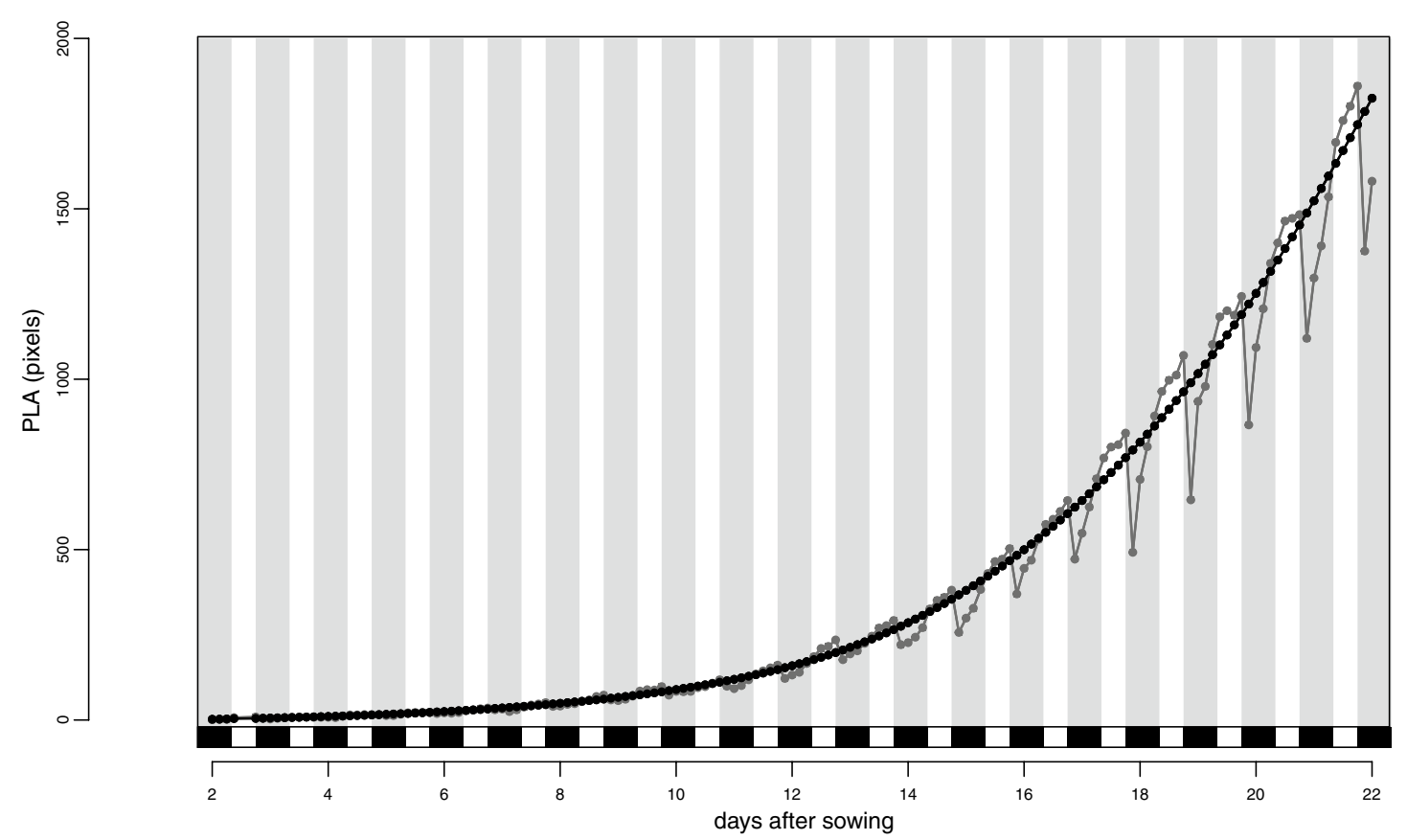

Fig. 5 Curve parameterisation of projected leaf area (PLA) for one replicate of genotype An-2. Grey line and filled squares show raw data, while black line and filled circles show fitted values; white and grey bars, projected in white and black on the X-axis, indicate the day-night cycle

changes through time and are different depending of the light intensity (Fig. 6b, c). Heritability of percentage change in PLA on the other hand is much more dynamic, with values shifting from 0.04 to 0.83 in the course of $6 \mathrm{~h}$ (Fig. 6d), emphasizing the importance of frequent measurements. In general the heritability was slightly lower at higher light intensity, probably due to reduced overall leaf movement (Fig. 4g, h). The most pronounced fluctuation is between day and night with heritability being much higher in the night than during the day. For spectral reflectance and growth curve traits the heritability also shows variation through time but in a less dynamic fashion, shifting over the course of several days (Additional file 7: Figure S4; Additional file 8: Figure S5).

\section{Discussion}

\section{Uniformity and reproducibility}

Uniformity and reproducibility of the Phenovator is essential if it is to be of any use. Although some design factors had a considerable main effect on the measurement (see Additional file 4: Data S1, Additional file 5: Data S2), the interaction of these design factors with genotype was very small and can be corrected for. The effect of the position within the image can be attributed to light gradients in the camera head, while the effect of experiment may be due to small accumulated differences which are collectively significant but individually minor [32]. The correction for design factors is achieved through the calculation of BLUEs for each genotype (Fig. 4). The ability to correct design factors greatly increased the signalto-noise ratio of the Phenovator, with signal being the genotypic effect and noise being unexplained phenotypic variation. This will be important when screening genetic mapping populations, as a larger contribution of the genotypic effect to the signal will increase the heritability of the measured trait. Finally, the estimation of the effect of experiment and its negligible interaction with genotype or other design factors allowed the combination of data from different experiments, greatly increasing the effective capacity of the system and the power of our statistical analysis.

\section{Phenomic data}

The only comparable system measuring photosynthetic and growth parameters is the GROWSCREEN FLUORO [23], which can phenotype up to 60 plants $\mathrm{h}^{-1}$ for growth and dark-adapted (maximum) PSII efficiency (Fv/Fm). Our system can measure the PLA of 1440 plants in $20 \mathrm{~min}$, and their light-adapted PSII efficiency, or operating efficiency $\left(\Phi_{\mathrm{PSII}}, \mathrm{Fq}^{\prime} / \mathrm{Fm}^{\prime}\right)$ [3], in less than an hour. The operating efficiency of $\Phi_{\mathrm{PSII}}$ directly relates to the rate of carbon fixation and ultimately growth and thus is physiologically more relevant than $\mathrm{Fv} / \mathrm{Fm}$ when assessing genotype performance in a range of conditions [16]. Using measurements of $\Phi_{\text {PSII }}$ we were able to determine differences in the rate of photosynthesis and estimate 


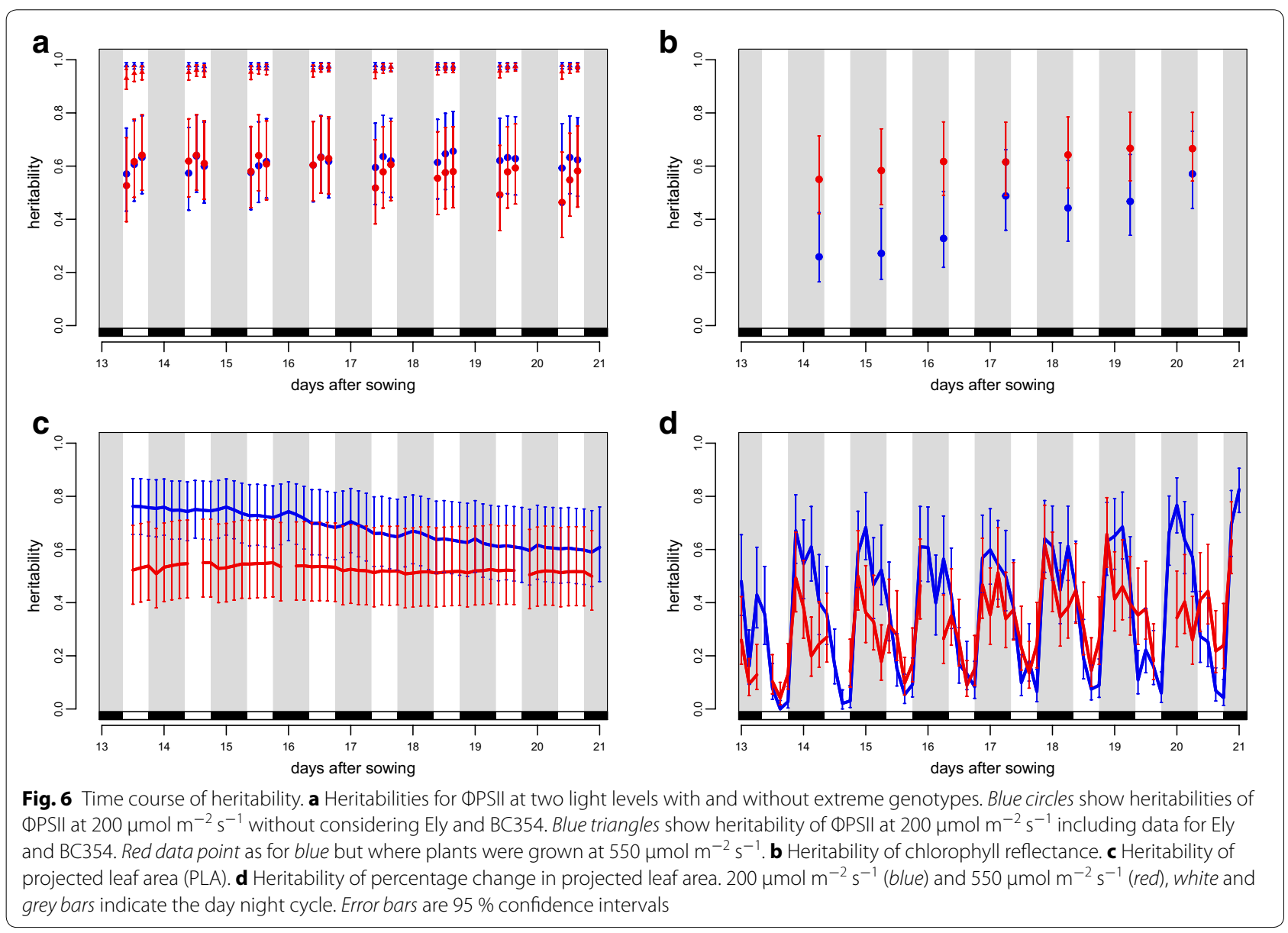

the genetic contribution to these differences for 20 genotypes of Arabidopsis grown at two light levels (Figs. 4a, b, 6). Of interest is the daily rise in $\Phi_{\text {PSII }}$ for all genotypes at $550 \mu \mathrm{mol} \mathrm{m} \mathrm{m}^{-2} \mathrm{~s}^{-1}$ with the exception of RIL BC354, which shows a daily decline in $\Phi_{\text {PSII }}$. The mutant allele of PDE237 (At4g30720), normally encoding an oxidoreductase/electron carrier residing in the chloroplast stroma [43], probably affects $\Phi_{\mathrm{PSII}}$ due to accumulated PSII damage during the day.

Using NIR light allowed us to measure PLA throughout the day and night without disturbing the photoperiod. Since one NIR measurement of all 1440 plants takes only 20 min we could measure all plants 72 times per day. This frequency exceeds that required to capture growth or leaf movement in most cases, but it could be valuable to capture rapid responses such as those induced upon water stress or disease infection. For measurements of growth or leaf movement under non-stressed conditions, imaging once every $3 \mathrm{~h}$ has proven to be sufficient (Figs. $4 \mathrm{~g}$, h, 5).

In addition to our priorities of measuring plant growth and photosynthesis we also measured the reflectance of individual plants at eight wavelengths of light. This made spectral imaging and estimating pigment content possible, which was also highly reproducible across experiments and genotypes. We were able to show a decline in chlorophyll reflectance when the plants were grown under high light conditions (Fig. 4c, d) which is expected according to literature [1].

\section{Heritability through time}

Using the phenotypic values for all traits across all genotypes we calculated the broad sense heritability of the different traits. As expected, heritability was trait specific, reflecting the genetic variation present for the trait. An unexpected finding was the amount this could vary through time. Daily fluctuations in heritability for some traits ranged from 0.04 to 0.83 (Fig. 6). As far as we know it is the first time this has been described in such detail, which is the consequence of imaging at such high frequency. We would never have detected this if images were taken at single or irregular time points per day. The magnitude and frequency of this variation in heritability was much greater than expected and strongly argues for 
high-frequency measurements. In the case of percentage change in PLA every $3 \mathrm{~h}$, reflecting leaf angle at different time points (Fig. 6b), the fluctuations in heritability show a diurnal pattern with a recurrent decline during the day under both light intensities. This may indicate the higher selection intensities present for leaf angle in light than in the dark, or alternatively a wider range of optima for leaf angle in the dark. In this case the measurement frequency was sufficient to capture changes in leaf movement using a simple difference method (the step change in PLA). This is apparent as an episodic, daily event. Note that this simple difference method captures the change in PLA due to leaf movement, but not that due to growth. When the measurements are viewed collectively, growth is nonetheless apparent (Fig. 5).

Two recent studies in Arabidopsis used high-throughput phenotyping to describe changing heritability through time. The first showed changes in the heritability for rosette compactness, which appears to increase linearly until the rosette has fully formed [46]. Two other traits, rosette area and circular area, showed fewer changes. The second study focused on root gravitropism [33]; after being reoriented by $90^{\circ}$, roots of seedlings were imaged every $2 \mathrm{~min}$ for $8 \mathrm{~h}$. The gravitropic response also showed a change in heritability through time. Interestingly some of the QTL underlying this changing heritability were time specific and only detectable for short periods.

Our results show both large and dynamic fluctuations in heritability due to changes in the relative contribution of genetic diversity to the traits at different time points (Fig. 6; Additional file 7: Figure S4; Additional file 8: Figure S5). The implications of this dynamic variation in heritability are wide ranging. For traits with such strong fluctuations in heritability, the time they are measured at will have a considerable impact on the extent of variation found. For crop breeding programs this could result in the fixation of alleles which may not be optimal for trait improvement. Screening when heritability is low will reduce the ability to detect genetic variation and the response of the germplasm to selection is likely to be curtailed [42]. This can lead to a waste of resources in large-scale breeding experiments. Awareness of the fluctuations in heritability can also be used to inform the breeder when the variation in phenotypes is most relevant. As shown by Moore et al. [33] the genetic loci responsible for the changing heritability can change through time. If fixation of a specific locus or set of loci is required, then identification of the time when they contribute most to phenotypic variance will result in more targeted breeding, and again, greater efficiency.
Awareness of the extent and time dependency of variation in heritability will thus maximise the return on investment in trait selection [4].

From an ecological and evolutionary perspective, stronger selection often results in reduced heritability [34], thus if the intensity of selection varies with developmental time, traits which contribute to fitness when selection is greatest are likely to show a reduction in heritability. While this will require further validation it illustrates the value of high-throughput phenotyping for generating insights into the genetic architecture of traits and the uses of such insights in the fields of breeding and evolutionary ecology.

\section{Conclusion: where next?}

The objective of our work has been to develop a high throughput phenotyping platform for photosynthesis $\left(\Phi_{\text {PSII }}\right)$ and growth. The rationale behind this is that phenotyping advances are essential for further rapid progress in plant genetics and breeding [15, 22, 37]. The choice of photosynthesis and growth was key, as they are both important traits with a complex polygenic architecture, and reliable high throughput phenotyping methods are needed if we are to mine natural variation or induced mutant libraries for these traits. Photosynthesis is of particular importance as it is the only major physiological trait not to have been directly bred for, and thus represents uncharted territory within which there may be considerable scope for crop improvement [14, 30]. In nature photosynthesis has been shaped by selection in environments where many resources are limited but the supply of fixed carbon is not usually a limiting factor for growth [26], while in agriculture, resources are more abundant and the supply of fixed carbon is often limiting [35]. Adaptations which evolved to increase survival in the wild, but reduce yield in an agricultural context, may be selectively removed [8]. For any such breeding program to be a success, there needs to be appropriate phenotyping [6]. We have proved this is possible for Arabidopsis, though the system we describe would be suitable for any species which forms a flat rosette and for seedlings of most other species. Besides their importance for crop improvement, high throughput phenotypers are essential for quantitative genetic studies such as QTL or GWA mapping. High throughput screening will aid forward genetics approaches for the identification of QTL and the genes responsible for the phenotypic differences in a population $[37,40]$. This is especially relevant when looking at natural accessions as such differences may represent adaptive alleles increasing fitness under specific 
environmental conditions [40]. Identification of such alleles is of interest for evolutionary biology and ecology, and to plant breeding as a source of genetic adaptations, which can be used to tailor crop varieties to specific conditions.

The stability and design of the system allowed the combination of data from multiple experiments, increasing the effective capacity beyond the 1440 plants which can fit in a single screen. The design is such that a range of environmental variables, such as temperature, humidity and nutrient availability, can be controlled both across and during experiments. To illustrate this flexibility we conducted one experiment at a higher light intensity. Such variation in the growth environment can be used to uncover hidden genetic variation not expressed under control conditions and identify genes important for adaptation to environmental fluctuations [19].

Phenomic data is also essential for the advance of the omics revolution. To put all the current omics technologies into context, whole plant phenotyping of morphological and physiological traits is necessary. Without such phenomic data the relevance of variation in gene expression, metabolite or protein abundance to plant performance is much more difficult to assess. The integration of all levels of omics data from gene expression to growth rate will allow a systems biology approach to be undertaken which should greatly further our understanding of plant biology [7, 24, 45]. Our data show how informative phenomics data can be, revealing, for example, how a basic genetic parameter such as heritability can vary through time. This insight is a direct result of the expanded throughput, and particularly, intensity of measurements. The level of accuracy and throughput of our system shows it to be ideally suited for screening large populations of plants thus allowing future quantitative genetic studies of photosynthesis, growth, and the response of these traits to a range of environmental perturbations in Arabidopsis or any rosette species, and thus explore a wide range of dynamic responses of plants, in detail, over time.

\section{Methods}

\section{Plant material and cultivation}

Unless otherwise stated all plants were grown as follows: seed was sown on wet filter paper and stratified for 6 days at $4{ }^{\circ} \mathrm{C}$. After stratification seed was sown directly on wet rockwool (www.grodan.com) which had been pre-soaked in a nutrient solution designed for Arabidopsis (see Additional file 9: Table S1 for composition). One seed was sown per rockwool block (system described in "System design" section). The growth conditions were as follows, $10 / 14 \mathrm{~h}$ day/night, irradiance normally $200 \mu \mathrm{mol} \mathrm{m}^{-2} \mathrm{~s}^{-1}$, and $550 \mu \mathrm{mol} \mathrm{m} \mathrm{m}^{-2} \mathrm{~s}^{-1}$ in the high light experiment, $20 / 18{ }^{\circ} \mathrm{C}$ day/night temperature, $70 \%$ relative humidity, and ambient $\mathrm{CO}_{2}$. Plants were irrigated daily with nutrient solution for $5 \mathrm{~min}$. In total 57 genotypes were screened across four experiments, see Additional file 2: Appendix S1 for details of genotype identity and number of replicates. The $\Phi_{\text {PSII }}$ estimates were compared with those of a MINI-PAM fluorometer (www.walz.com) to validate the measurements and no significant differences were found.

\section{Measurement protocols}

$\Phi_{\text {PSII }}$ was measured daily, 1,4 and $7 \mathrm{~h}$ into the photoperiod. This was considered sufficient to document any variations in the phenotype and allowed time for other measurements such as NIR, which was measured every $3 \mathrm{~h}$.

\section{Statistical analysis}

\section{Variance components}

The importance of several design factors was assessed by fitting the following mixed model for each trait and timepoint using asreml-R [5]:

$$
\begin{aligned}
Y= & \mu+C+G+\operatorname{Exp}+\text { Basin }+x+y+\text { TablePosition } \\
& +x_{\text {within }}+y_{\text {within }}+G \times \text { Exp }+ \text { Exp } \\
& \times\left(\text { Basin }+x+y+\text { TablePosition }+x_{\text {within }}+y_{\text {within }}\right) \\
& + \text { Exp } \times \text { Basin } \times G+\operatorname{Exp} \times G \times\left(x_{\text {within }}+y_{\text {within }}\right) \\
& +R(\text { Error })
\end{aligned}
$$

where $\mu$ is the overall mean, and G, Exp and Basin are the factors for respectively genotype, experiment and basin. The factor $\mathrm{C}$ represents check-genotypes that were not included in subsequent analyses, but included in the mixed model in order to better estimate the variance components; it has one level for each check-genotype and one additional level representing all other genotypes. All terms except $\mu$ and $C$ are defined as random effects. For traits and time-points that were only present in a single experiment, all terms involving Exp were dropped from the model. Spatial variability was modelled by the factors $x, y$, TablePosition, $x_{\text {within }}$ and $y_{\text {within }}$ which represent respectively rows, columns, table (camera/image) position and within image rows and columns. While $\mathrm{x}$ and y model the coordinates across the whole platform, $x_{\text {within }}$ and $y_{\text {within }}$ model the spatial effects within images of 12 plants $(3 \times 4)$. A more detailed description of all design factors is given in Additional file 3: Appendix S2. 


\section{Genotypic means}

Genotypic means used in Fig. 4 were calculated as the best linear unbiased estimators (BLUEs) for genotype, using a mixed model identical to Eq. (1) but with genotype as fixed effect.

\section{Heritability estimates}

Defining and estimating heritability in the context of a mixed model as defined by Eq. (1) is known to be difficult, since not only the residual error contributes to the environmental variance (the generalized heritability proposed in Oakey et al. [36] concerns line heritability and not the (plot level) heritability $\sigma_{G}^{2} /\left(\sigma_{G}^{2}+\sigma_{E}^{2}\right)$, which is of interest here). To obtain more interpretable and commonly used heritability estimates we therefore performed classical analysis of variance (ANOVA) for the linear model with (fixed) effects for genotype, basin nested within experiment, and within image $x_{\text {within }}$ and $y_{\text {within }}$ coordinates. This included the most important main effects identified by the mixed model analysis described above; the fact that the interactions of design factors with genotype were small, justifies the effects being fixed here. The genetic and environmental variance were estimated by respectively $(M S(G)-M S(E)) / \bar{r}$ and $\mathrm{MS}(\mathrm{E})$, where MS(G) and MS(E) are the mean sums of squares for genotype and residual error [28, 31]. Broad sense heritability was then estimated by the ratio of estimated genetic variance over the sum of estimated genetic and environmental variance. To facilitate direct comparison, heritability was estimated using 20 genotypes which were screened under both light conditions (see Additional file 2: Appendix S1 for details on the genotypes used).

\section{Growth curve characterisation}

PLA was measured throughout each experiment from NIR images and the masks generated from the $\Phi_{\text {PSII }}$ images, a total of 11 images per day. In order to summarize these data and estimate growth rates from repeated plant-size measurements, a flexible curve was fitted to the data for each plant. We used P-splines as a flexible semiparametric description of the curves [10]. P-splines are penalized B-splines resulting in smooth piecewise polynomial curves. For the implementation in the context of this paper we used the R package mgcv [44] with the function gam with its option for P-splines. Fitted curves and addition growth parameters are plotted in Fig. 5 and Additional file 10: Figure S3. The (empirical) slope at all time points is calculated directly from the fitted values of the curve. Relative growth rates can be calculated based on the raw data series. However, for fluctuating time series growth rates are more reliable when a smooth curve is base of their calculation (Additional file 10: Figure S3).

\section{Additional files}

Additional file 1: Figure S1. Print screen showing analysis software. The top left panel shows all 120 imaging positions in green with the positions of the four replicates highlighted in yellow. These images of 12 plants are shown in the top row of pictures headed Rep A to Rep D. The plant which corresponds to the genotype being analysed is surrounded by a red box in each image. This plant is cut from the image using a mask level set in the control panel which is shown at the bottom of the image. The resulting image is thing shown by the middle row of pictures. Note this shows a pixel map of DPSIl distribution. This pixel map is then plotted as a histogram for each image in the last row of pictures.

Additional file 2: Appendix S1. Overview of experiments conducted and genotypes used. A list of aliases and geographical origins is also included as well as the number of replicates sown and the experiments each genotype was included in.

Additional file 3: Appendix S2. Description of design factors described in Data S1 and Data S2, calculated across three experiments at $200 \mu \mathrm{mol} \mathrm{m} \mathrm{s}^{-1}$ and across a single experiment at $550 \mu \mathrm{mol} \mathrm{m} \mathrm{m}^{-2} \mathrm{~s}^{-1}$. See Appendix S1 for further details on experiments and number of replicates used.

Additional file 4: Data S1. Variances of all design factors and interactions for each trait at $200 \mu \mathrm{mol} \mathrm{m} \mathrm{m}^{-2} \mathrm{~s}^{-1}$. Separate excel sheets correspond to different traits measured. Raw data and graphs of variance components through time are shown on each excel sheet.

Additional file 5: Data S2. Variances of all design factors and interactions for each trait at $550 \mu \mathrm{mol} \mathrm{m} \mathrm{m}^{-2} \mathrm{~s}^{-1}$. Separate excel sheets correspond to different traits measured. Raw data and graphs of variance components through time are shown on each excel sheet.

Additional file 6: Figure S2. Phenotypic variation in spectral reflectance at eight wavelengths. Phenotypic diversity in twenty Arabidopsis genotypes grown at $200 \mu \mathrm{mol} \mathrm{m} \mathrm{m}^{-2} \mathrm{~s}^{-1}$ light intensity $(\mathrm{a}, \mathrm{c}, \mathrm{e}, \mathrm{g}, \mathrm{i}, \mathrm{k}, \mathrm{m}, \mathrm{O})$, and $550 \mu \mathrm{mol} \mathrm{m} \mathrm{m}^{-2} \mathrm{~s}^{-1}$ light intensity (b, d, f, h, j, l, n, p). Wavelength assessed is indicated on the $y$ axis. All data points are genotypic means (BLUEs), combining observations on replicates from different experiments into one representative value for each genotype at each time point. Six genotypes, An-2 (yellow circles), BC354 (purple circles), Bur-0 (green squares), Col-0 (black triangles), Ely (red circles) and Ts-1 (blue squares) are indicated in colour. Error bars have been excluded for clarity, the significance of between genotype differences is apparent from the heritability estimates in Figure $\mathrm{S4}$

Additional file 7: Figure S4. Time course of Heritability for spectral reflectance at eight wavelengths. (a) $480 \mathrm{~nm}$, (b) $532 \mathrm{~nm}$, (c) $550 \mathrm{~nm}$, (d) $570 \mathrm{~nm},(\mathrm{e}) 660 \mathrm{~nm},(\mathrm{f}) 700 \mathrm{~nm},(\mathrm{~g}) 750 \mathrm{~nm}$, and (h) $790 \mathrm{~nm} .200 \mu \mathrm{mol} \mathrm{m}{ }^{-2}$ $\mathrm{s}^{-1}$ (blue) and $550 \mu \mathrm{mol} \mathrm{m} \mathrm{s}^{-1}$ (red), white and grey bars indicate the day night cycle. Error bars are $95 \%$ confidence intervals.

Additional file 8: Figure S5. Time course of heritability of growth curve parameters for plants grown at $200 \mu \mathrm{mol} \mathrm{m} \mathrm{m}^{-2} \mathrm{~s}^{-1}$ light intensity. (a) Projected leaf area (PLA) from near infrared (NIR) measurements. (b) Data from (a) fitted to a curve. (c) The empirical slope of the growth curve and (d) the relative growth rate. White and grey bars indicate the day/night cycle. Error bars are 95\% confidence intervals.

Additional file 9: Table S1. Nutrient solution composition.

Additional file 10: Figure S3. Phenotypic variation in growth curve parameters. Phenotypic diversity in twenty Arabidopsis genotypes grown at $200 \mu \mathrm{mol} \mathrm{m} \mathrm{m}^{-2} \mathrm{~s}^{-1}$ light intensity. (a) Projected leaf area (PLA) from near infrared (NIR) measurements. (b) Data from (a) fitted to a curve. (c) The empirical slope of the growth curve and (d) the relative growth rate. All data points are genotypic means (BLUEs), combining observations on replicates from different experiments into one representative value for each genotype at each time point. Six genotypes, An-2 (yellow circles), BC354 (purple circles), Bur-0 (green squares), Col-0 (black triangles), Ely (red circles) and Ts-1 (blue squares) are indicated in colour. Error bars have been excluded for clarity, the significance of between genotype differences is apparent from the heritability estimates in Figure S5. 


\section{Abbreviations}

ANOVA: analysis of variance; BLUEs: best linear unbiased estimators; Chl: chlorophyll reflectance; Fv/Fm: dark-adapted (maximum) PSIl efficiency; FWHM: full width at half maximum; GWA: genome wide association; LED: light emitting diodes; NIR: near infrared; PLA: projected leaf area; PSII: photosystem II; QTL: quantitative trait loci; RIL: recombinant inbred line; $\Phi_{\text {PSII }}$ : light-adapted PSII efficiency, or PSIl operating efficiency.

\section{Authors' contributions}

Conception and design of phenotyper HJ, JH, JFHS, MGMA, RvdS and PJF. Construction of the phenotyper $\mathrm{HJ}$ and RvdS. Programming of control and analysis software RvdS. Testing of phenotyper HJ, JH, JFHS, RvdS and PJF. Conducted experiments PJF. Analysed data PJF, WK and SKS. Wrote the paper PJF, JH and MGMA with comments from all other authors. All authors read and approved the final manuscript.

\section{Author details}

${ }^{1}$ Laboratory of Genetics, Wageningen University, Wageningen, The Netherlands. ${ }^{2}$ Horticulture and Production Physiology, Wageningen University, Wageningen, The Netherlands. ${ }^{3}$ Biometris, Wageningen University and Research Centre, Wageningen, The Netherlands. ${ }^{4}$ Greenhouse Horticulture, Wageningen University and Research Centre, Wageningen, The Netherlands. ${ }^{5}$ Present Address: Department of Plant Breeding and Genetics, Max Planck Institute for Plant Breeding Research, Cologne, Germany. ${ }^{6}$ Present Address: PhenoVation BV, Wageningen, The Netherlands. ${ }^{7}$ Present Address: Adviesbureau JFH Snel, Wageningen, The Netherlands.

\section{Acknowledgements}

We thank Charles Neris Moreira for the use of his phosphorus deficient plant for Fig. 1 and help with planting experiments. Eltje Groendijk and Gradus Leenders for technical assistance in construction. Gerrit Stunnenberg and Taede Stoker for help in running the experiments. Roxanne van Rooijen for her comments on the draft manuscript and Bas Zwaan for insightful discussions surrounding heritability, its uses and significance. This work was financially supported by an ALW/TTI-Green Genetics grant from the Netherlands Organisation for Scientific Research (NWO); by the Dutch Technology Foundation STW, which is part of the NWO, and which is partly funded by the Ministry of Economic Affairs; and by the DROught-tolerant yielding PlantS (DROPS) project, which received funding from the European Community's Seventh Framework Programme (FP7/2007-2013) under grant agreement 244374.

\section{Competing interests}

JFHS, HJ and RvdS left Wageningen University on July 31, 2014. JFHS owns an independent consultancy 'Adviesbureau JFH Snel'on photosynthesis monitoring in greenhouse horticulture. $\mathrm{HJ}$ and RvdS run a company, Phenovation, which sells phenotyping systems in which they are both shareholders. In all cases the move to private sector occurred after the research described in this manuscript was conducted. All other authors declare no competing interests.

Received: 28 October 2015 Accepted: 25 January 2016

Published online: 15 February 2016

\section{References}

1. Anderson J, Chow W, Park Y-I. The grand design of photosynthesis: acclimation of the photosynthetic apparatus to environmental cues. Photosynth Res. 1995;46:129-39.

2. Arvidsson S, Pérez-Rodríguez P, Mueller-Roeber B. A growth phenotyping pipeline for Arabidopsis thaliana integrating image analysis and rosette area modeling for robust quantification of genotype effects. New Phytol. 2011;191:895-907.

3. Baker NR. Chlorophyll fluorescence: a probe of photosynthesis in vivo. Annu Rev Plant Biol. 2008;59:89-113.

4. Brown TB, Cheng R, Sirault XRR, et al. TraitCapture: genomic and environment modelling of plant phenomic data. Curr Opin Plant Biol. 2014;18:73-9.

5. Butler D, Cullis BR, Gilmour A, et al. ASReml-R reference manual. Brisbane: Queensland Department of Primary Industries and Fisheries; 2007.
6. Cabrera-Bosquet L, Crossa J, Von Zitzewitz J, et al. High-throughput phenotyping and genomic selection: the frontiers of crop breeding converge. J Integr Plant Biol. 2012;54:312-20.

7. Chen M, Hofestädt R, Chen D, et al. Bridging genomics and phenomics. In: Chen M, Hofestädt R, editors. Approaches in integrative bioinformatics. Berlin, Heidelberg: Springer; 2014. p. 299-333.

8. Denison RF. Darwinian agriculture: how understanding evolution can improve agriculture. Princeton: Princeton University Press; 2012.

9. Eberhard S, Finazzi G, Wollman F-A. The dynamics of photosynthesis. Annu Rev Genet. 2008;42:463-515.

10. Eilers PHC, Marx BD. Flexible smoothing with $B$-splines and penalties. Stat Sci. 1996;11:89-102.

11. El-Lithy ME, Rodrigues GC, Van Rensen JJS, et al. Altered photosynthetic performance of a natural Arabidopsis accession is associated with atrazine resistance. J Exp Bot. 2005;56:1625-34.

12. Fatichi S, Leuzinger S, Körner C. Moving beyond photosynthesis: from carbon source to sink-driven vegetation modeling. New Phytol. 2014;201:1086-95.

13. Finkel E. With 'phenomics', plant scientists hope to shift breeding into overdrive. Science. 2009;325:380-1.

14. Flood PJ, Harbinson J, Aarts MGM. Natural genetic variation in plant photosynthesis. Trends Plant Sci. 2011;16:327-35.

15. Furbank RT, Tester M. Phenomics - technologies to relieve the phenotyping bottleneck. Trends Plant Sci. 2011;16:635-44.

16. Genty B, Briantais J-M, Baker NR. The relationship between the quantum yield of photosynthetic electron transport and quenching of chlorophyll fluorescence. Biochim Biophys Acta (BBA) Gen Subj. 1989:990:87-92.

17. Genty B, Meyer S. Quantitative mapping of leaf photosynthesis using chlorophyll fluorescence imaging. Funct Plant Biol. 1995;22:277-84.

18. Gibeaut DM, Hulett J, Cramer GR, et al. maximal biomass of arabidopsis thaliana using a simple, low-maintenance hydroponic method and favorable environmental conditions. Plant Physiol. 1997;115:317-9.

19. Gibson G, Dworkin I. Uncovering cryptic genetic variation. Nat Rev Genet. 2004:5:681-90.

20. Gitelson AA, Gritz Y, Merzlyak MN. Relationships between leaf chlorophyll content and spectral reflectance and algorithms for nondestructive chlorophyll assessment in higher plant leaves. J Plant Physiol. 2003; 160:271-82.

21. Houle D. Numbering the hairs on our heads: the shared challenge and promise of phenomics. Proc Natl Acad Sci. 2010;107:1793-9.

22. Houle D, Govindaraju DR, Omholt S. Phenomics: the next challenge. Nat Rev Genet. 2010;11:855-66.

23. Jansen M, Gilmer F, Biskup B, et al. Simultaneous phenotyping of leaf growth and chlorophyll fluorescence via GROWSCREEN FLUORO allows detection of stress tolerance in Arabidopsis thaliana and other rosette plants. Funct Plant Biol. 2009;36:902-14.

24. Joyce AR, Palsson BO. The model organism as a system: integrating 'omics' data sets. Nat Rev Mol Cell Biol. 2006;7:198-210.

25. Kahlau S, Bock R. Plastid transcriptomics and translatomics of tomato fruit development and chloroplast-to-chromoplast differentiation: chromoplast gene expression largely serves the production of a single protein. Plant Cell Online. 2008;20:856-74.

26. Körner C. Growth controls photosynthesis-mostly. Nova Acta Leopold. 2013;114:273-83.

27. Korte A, Farlow A. The advantages and limitations of trait analysis with GWAS: a review. Plant Methods 2013;9:1-9.

28. Kruijer W, Boer M, Malosetti M, et al. Marker-based estimation of heritability in immortal populations. Genetics. 2015;199:1-20.

29. Leister D, Varotto C, Pesaresi P, et al. Large-scale evaluation of plant growth in Arabidopsis thaliana by non-invasive image analysis. Plant Physiol Biochem. 1999;37:671-8.

30. Long SP, Zhu X-G, Naidu SL, et al. Can improvement in photosynthesis increase crop yields? Plant Cell Environ. 2006;29:315-30.

31. Lynch M, Walsh B. Genetics and analysis of quantitative traits. Sunderland: Sinauer; 1998.

32. Massonnet C, Vile D, Fabre J, et al. Probing the reproducibility of leaf growth and molecular phenotypes: a comparison of three Arabidopsis accessions cultivated in ten laboratories. Plant Physiol. 2010;152:2142-57. 
33. Moore CR, Johnson LS, Kwak I-Y, et al. High-throughput computer vision introduces the time axis to a quantitative trait map of a plant growth response. Genetics. 2013;195:1077-86.

34. Mousseau TA, Roff DA. Natural selection and the heritability of fitness components. Heredity. 1987;59:181-97.

35. Murchie EH, Niyogi KK. Manipulation of photoprotection to improve plant photosynthesis. Plant Physiol. 2011;155:86-92.

36. Oakey H, Verbyla A, Pitchford W, et al. Joint modeling of additive and non-additive genetic line effects in single field trials. Theor Appl Genet. 2006;113:809-19.

37. Pieruschka R, Poorter H. Phenotyping plants: genes, phenes and machines. Funct Plant Biol. 2012;39:813-20.

38. Simon M, Loudet O, Durand S, et al. Quantitative trait loci mapping in five new large recombinant inbred line populations of Arabidopsis thaliana genotyped with consensus single-nucleotide polymorphism markers. Genetics. 2008;178:2253-64.

39. Skirycz A, Vandenbroucke K, Clauw P, et al. Survival and growth of Arabidopsis plants given limited water are not equal. Nat Biotechnol. 2011;29:212-4.
40. Tisné S, Serrand Y, Bach L, et al. Phenoscope: an automated large-scale phenotyping platform offering high spatial homogeneity. Plant J. 2013;74:534-44

41. Van Kooten $\mathrm{O}$, Snel JH. The use of chlorophyll fluorescence nomenclature in plant stress physiology. Photosynth Res. 1990;25:147-50.

42. Visscher PM, Hill WG, Wray NR. Heritability in the genomics era-concepts and misconceptions. Nat Rev Genet. 2008;9:255-66.

43. Vlad D, Rappaport F, Simon M, et al. Gene transposition causing natural variation for growth in Arabidopsis thaliana. PLoS Genet. 2010;6:e1000945

44. Wood SN. Fast stable restricted maximum likelihood and marginal likelihood estimation of semiparametric generalized linear models. J R Stat Soc Ser B (Stat Methodol). 2011;73:3-36.

45. Yuan JS, Galbraith DW, Dai SY, et al. Plant systems biology comes of age. Trends Plant Sci. 2008;13:165-71.

46. Zhang $X$, Hause RJ, Borevitz JO. Natural genetic variation for growth and development revealed by high-throughput phenotyping in Arabidopsis thaliana. G3: Genes|Genomes|Genetics. 2012;2:29-34.

\section{Submit your next manuscript to BioMed Central and we will help you at every step:}

- We accept pre-submission inquiries

- Our selector tool helps you to find the most relevant journal

- We provide round the clock customer support

- Convenient online submission

- Thorough peer review

- Inclusion in PubMed and all major indexing services

- Maximum visibility for your research

Submit your manuscript at www.biomedcentral.com/submit
(O) BioMed Central 